GASTRIC CANCER

\section{Dysregulation of RNA editing in gastric cancer}

New research published in

Gastroenterology has shown that gastric cancers have increased rates of RNA editing dysfunction - so-called 'misediting' — mediated by altered expression of the RNA-editing enzymes ADAR1 and ADAR2.

Gastric cancer is a common and highly lethal malignancy, estimated to be the third leading cause of cancer-related mortality. Most molecular studies of gastric cancer to date have focused on DNA alterations associated with the disease. However, evidence from studies of other cancers suggests that post-transcriptional RNA editing might also contribute to oncogenic mutations,

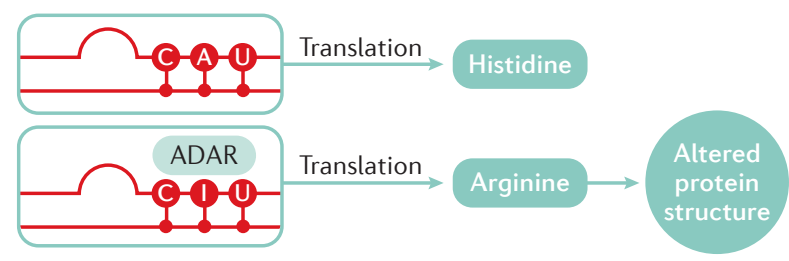

Representation of a structural protein change induced by RNA editing. which are missed when only DNA is examined. Deamination of adenosine to inosine (A-to-l editing), catalysed by ADAR1 (also known as double-stranded RNA-specific adenosine deaminase) and ADAR2 (also known as double-stranded RNA-specific editase 1), is the most common form of RNA editing in humans. Importantly, inosine is recognized as guanosine by translational machinery, leading to the potential introduction of mutations.

"We leveraged high-throughput transcriptome sequencing of primary gastric cancer tissues and cell lines to dissect the relationship between RNA editing and cancer progression and prognosis," explains author Leilei Chen. Compared with paired healthy gastric tissue samples, the researchers found substantial hypoediting and hyperediting in gastric cancer samples obtained from 14 patients. In a cohort comprising samples from 185 gastric tumours and 89 regions of healthy gastric tissue, Chen and colleagues found that tumour samples overexpressed ADAR1, whereas ADAR2 was downregulated. Notably, patients with tumours with combined ADAR1 overexpression and ADAR2 downregulation had the poorest prognosis, findings that were validated in a separate cohort.

To probe the association between gastric cancer pathogenesis and RNA editing, the investigators examined the editing frequencies of validated ADAR-specific sites in normal gastric tissue, gastric tissue with premalignant intestinal metaplasia and gastric tumour tissue. Progression from healthy to tumorous tissue was associated with increased editing of ADAR1-specific sites, whereas editing of ADAR2-specific sites decreased.

"Our study highlights a major role for RNA editing in gastric cancer and its progression," comments Chen. "Our findings also suggest new therapeutic opportunities, through ADAR1 enzymatic inhibition or the potential restoration of ADAR2 activity."

Hugh Thomas

ORIGINAL ARTICLE Chan, T. H. et al. ADARmediated RNA editing predicts progression and prognosis of Gastric Cancer. Gastroenterology http://dx.doi.org/10.1053/j.gastro.2016.06.043 (2016) 